\title{
A Vector Potential implementation for Smoothed Particle Magnetohydrodynamics
}

\author{
Federico A. Stasyszyn, Detlef Elstner \\ Leibniz-Institut für Astrophysik Potsdam (AIP), An der Sternwarte 16, 14482, Postdam, Germany
}

\begin{abstract}
The development of smooth particle magnetohydrodynamic (SPMHD) has significantly improved the simulation of complex astrophysical processes. However, the preservation the solenoidality of the magnetic field is still a severe problem for the MHD. A formulation of the induction equation with a vector potential would solve the problem. Unfortunately all previous attempts suffered from instabilities. In the present work, we evolve the vector potential in the Coulomb gauge and smooth the derived magnetic field for usage in the momentum equation. With this implementation we could reproduce classical test cases in a stable way. A simple test case demonstrates the possible failure of widely used direct integration of the magnetic field, even with the usage of a divergence cleaning method.
\end{abstract}

Keywords: Magnetohydrodynamics - Astrophysics - methods: Smooth Particle Hydrodynamics

\section{Introduction}

In recent years, not only the presence but the morphology of magnetic fields in galaxies has been determined [2]. It represents a huge scientific challenge, as this is a new opportunity to understand how the magnetic field is related to the astrophysical hosts, their history and properties.

A possible explanation for the magnetic field amplification is the action of a dynamo driven by turbulence and large scale gas motions $[23,1,19]$, where the unknown initial seed field is washed out by the turbulent character of the flow. Numerical simulations of evolving galaxies should help to understand the main properties of the magnetic field amplification with the observed morphology.

The success of cosmological simulations using SPH methods motivates the application of that technique also for the MHD case [10]. The direct implementation of the induction equation with the magnetic field unfortunately suffers from the preservation of solenoidality. The artificial growth of $\nabla \cdot \mathbf{B}$ in these schemes is usually reduced by a more or less artificial cleaning of $\mathbf{B}$. The direct integration of $\mathbf{B}$ with or without cleaning may lead to unrealistic numerical growth of the magnetic field as it occurs in the example described in section 3 or in Kotarba et al. [13]. There is no $\nabla \cdot \mathbf{B}=0$ preserving scheme known for SPMHD integrating the magnetic field $\mathbf{B}$ directly from the induction equation. Changing the integration variable from the magnetic field to the vector potential $\mathbf{A}$ with $\mathbf{B}=\nabla \times \mathbf{A}$ solves the problem in a natural way. A vector potential formulation in SPH was previous studied in detail by Price [17]. The implementation was working for one and two dimensional problems, but failed in three dimensions.

In the following sections we present an application of the vector potential in SPMHD, which overcomes the previous problems. In section 2 we shortly describe our implementation, followed by the analysis of some test cases in section 3. Finally we discuss possible implications in section 4 and we present our conclusions in section 5 .

Email addresses: fstasys@aip.de (Federico A. Stasyszyn), delstner@aip.de (Detlef Elstner) 


\section{SPH Implementation}

Throughout this work we will use the SPMHD version of Gadget-3 [20], where the ideal MHD is solved following the induction equation in the form

$$
\frac{\mathrm{d} \mathbf{B}}{\mathrm{d} t}=(\mathbf{B} \cdot \nabla) \mathbf{v}-\mathbf{B}(\nabla \cdot \mathbf{v})
$$

in which, we assume the $\nabla \cdot \mathbf{B}=0$ constraint is valid, by taking special care on reducing it [20, 22].

Price [17] studied carefully the possible SPH vector potential formulation, we follow it and use it as a starting point. The definition of the magnetic field and the evolution of the vector potential can be summarized as follows:

$$
\begin{aligned}
\mathbf{B} & =\nabla \times \mathbf{A} \\
\frac{\mathrm{d} \mathbf{A}}{\mathrm{d} t} & =\mathbf{v} \times \nabla \times \mathbf{A}+(\mathbf{v} \cdot \nabla) \mathbf{A}-\nabla \phi
\end{aligned}
$$

where $\phi$ is an arbitrary scalar representing the freedom to choose a special gauge. There is the freedom of choosing different gauges for each time step if desired to improve the numerics, but keeping track of a proper $\phi$ evolution of a given particular gauge.

In tensor form the components of Eq. (3) simplify to

$$
\frac{\mathrm{d} A_{i}}{\mathrm{~d} t}=v^{j} \frac{\partial A_{j}}{\partial x^{i}}-\frac{\partial \phi}{\partial x^{i}}
$$

where $i, j$ are component indexes and summation over double indices is used.

In the SPH framework this equation is written as follows,

$$
\frac{\mathrm{d} A_{a}^{i}}{\mathrm{~d} t}=\frac{f_{a}}{\rho_{a}}\left[\sum_{b=1}^{N}-m_{b}\left(\phi_{a b}^{i}-v_{a}^{j} A_{a b}^{j}\right) \partial W_{a b}^{i}\right]
$$

where $a, b$ are particle indexes, $f_{a}$ is the correction factor that arises from the use of variable smoothing lengths, the $A_{a b}^{j}$ is the difference between the potential of neighboring particles and $\partial W_{a b}^{i}$ is the kernel gradient operator between particles (for more details refer to Dolag and Stasyszyn [10]).

As we mentioned before, the gauge choice does not manifest in the magnetic field, but in the evolution of the vector potential. For example, if we use the Coulomb gauge, which means $\nabla \cdot \mathbf{A}=0$ for all points of space and time, we have to take care of fulfilling this requirement. Therefore, we face a similar problem as keeping $\nabla \cdot \mathbf{B}=0$, that has already been extensively studied [20,22]. We take a similar approach, using a cleaning scheme [9] originally thought to lower the $\nabla \cdot \mathbf{B}$ errors, but applied to $\mathbf{A}$ in order to ensure $\nabla \cdot \mathbf{A}=0$. The solution of the problem is equivalent to choose a modified pseudo-Lorenz or velocity gauge [12, 6], with an additional damping term. Note, that keeping $\nabla \cdot \mathbf{A}=0$ will also simplify the calculation of the diffusion terms for the non-ideal MHD equations. The evolution of the gauge is achieved through following equations

$$
\frac{\mathrm{d} \phi}{\mathrm{d} t}=-c_{h}^{2} \nabla \cdot \mathbf{A}-c_{h} \frac{\phi}{h}-\frac{\nabla \cdot \mathbf{v} \phi}{2}
$$

where $c_{h}$ is the characteristic signal velocity, $h$ is the smoothing length and we add the final term, introduced by Tricco and Price [22] that takes into account compression or expansion of the fluid. Tricco and Price [22] found that this additional term, improves conservation of energy and in particular for the divergence cleaning is crucial the symmetrization of the SPH operators. In our case we use a "differential" nonsymmetric SPH operator and we do not apply any limiter as in Stasyszyn et al. [20], and seems sufficient to achieve stability. However, when coupling the energy evolution using a symmetric operator can improve the energy conservation.

Therefore the gauge evolution in SPH form writes as Eq. (6), and the differential operators takes the form for the divergence case as:

$$
\nabla \cdot \mathbf{A}=\frac{f_{a}}{\rho_{a}}\left[\sum_{b=1}^{N} m_{b} A_{a b}^{i} \partial W_{a b}^{i}\right]
$$




\subsection{Lorentz Force}

The coupling of the magnetic field and the momentum equation is done by calculating $\mathbf{B}$ from the potential and then applying it to the force equation using the magnetic stress tensor [15]

$$
M^{i j}=\left(\frac{B^{i} B^{j}}{\mu_{0}}-\frac{1}{2 \mu_{0}}|\mathbf{B}|^{2} \delta^{i j}\right) .
$$

which modifies the momentum equation by adding the term

$$
\left(\frac{\mathrm{d} v^{i}}{\mathrm{~d} t}\right)_{m a g}=\frac{1}{\rho}\left(\frac{\partial M^{i j}}{\partial x^{j}}-B^{i} \frac{\partial B^{j}}{\partial x^{j}}\right)
$$

The last term subtracts $\nabla \cdot \mathbf{B}$ errors which still occur here by the numerical approximation of the curl operator. It stabilizes the numerical scheme [4]. The use of this set of equations corresponds to the hybrid approach described by Price [17] and is equivalent at the force treatment described in Dolag and Stasyszyn [10] and subsequent works.

In order to further stabilize the scheme $\mathbf{B}$ we smooth magnetic field $\mathbf{B}$ before it is applied in the calculation of the Lorentz force. In contrast to the implementation presented by Dolag and Stasyszyn [10], we do not volume weight the smoothing operation, because it generates instabilities in low density regions. We smooth the field of the neighboring particles using only the SPH kernel computing

$$
B_{a}^{i}=\frac{\sum_{b} B_{b}^{i} W_{a b}}{\sum_{b} W_{a b}}
$$

Note that this additional step does not introduce any artificial dissipation for the induction process, because we do not change the time evolution of the vector potential. We use the same switches and the magnetic signal velocity described in Dolag and Stasyszyn [10]. To summarize, we calculate a B from the potential A, afterward we smooth the magnetic field and calculate the corresponding forces, using the same numerical corrections from previous implementations.

\subsection{Diffusion}

It is straightforward to implement a diffusion of the magnetic field through the potential. Taking care of keeping $\nabla \cdot \mathbf{A}=0$, we may simply write the diffusion term as

$$
\left(\frac{\mathrm{d} \mathbf{A}}{\mathrm{d} t}\right)_{d i f f}=\eta_{i} \nabla^{2} \mathbf{A}
$$

Note that, this formulation allows a spatial dependent dissipation $\eta$. The Laplacian calculation as described has previously been successfully implemented for spatially independent diffusion of the magnetic field [3, 18], and has been proven to be useful handling possible numerical instabilities, and is written in $\mathrm{SPH}^{1}$

\section{Testing the method}

We first show a simple kinematic test, where the direct integration of the magnetic field fails in contrast to the vector potential formulation of the induction equation. In order to apply this formalism for astrophysical problems, the non-linear effects due to feedback from the magnetic field on the dynamics have to be successfully treated in different environments. Therefore, we present the classic shock tube from Brio and $\mathrm{Wu}[7]$ and the more complex example of the Orszag and Tang [14] vortex with the vector field implementation. We always perform the tests in 3 dimensions, because previous attempts [17] failed particularly in those cases.

${ }^{1}$ Eq. 3 in Bonafede et al. [3] as

$$
\left(\frac{\mathrm{d} A_{i}}{\mathrm{~d} t}\right)_{d i f f}=2 \eta_{i} \rho_{a}\left[\sum_{b=1}^{N} \frac{m_{b}}{\hat{\rho}_{i j}^{2}} A_{a b}^{i} \frac{\mathrm{r}_{i j}}{\left|\mathrm{r}_{\mathrm{ij}}\right|} \partial W_{a b}^{i}\right]
$$



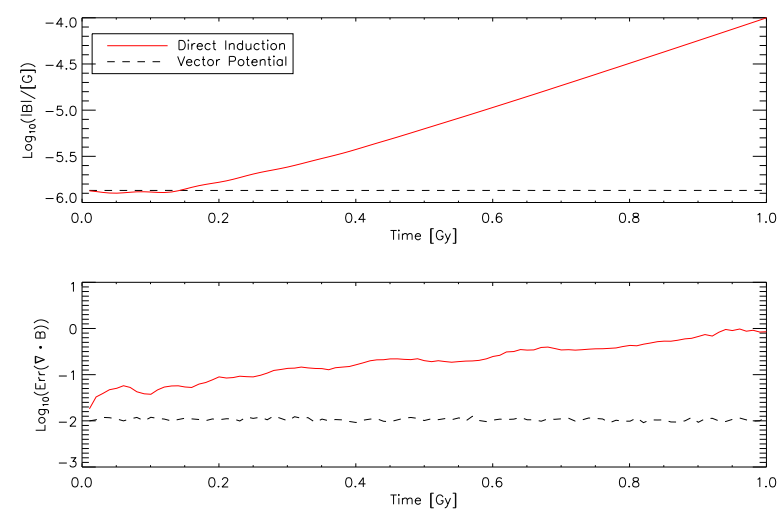

Figure 1: The upper panel shows the time evolution of the average magnetic field strength of the differentially rotating disk. We show the evolution of the vector potential implementation (dashed-black line) and of the direct integration (solid-red line). The lower panel shows the mean evolution of the divergence error that accumulates over time for the direct integration and stay constant in the case of the vector potential.
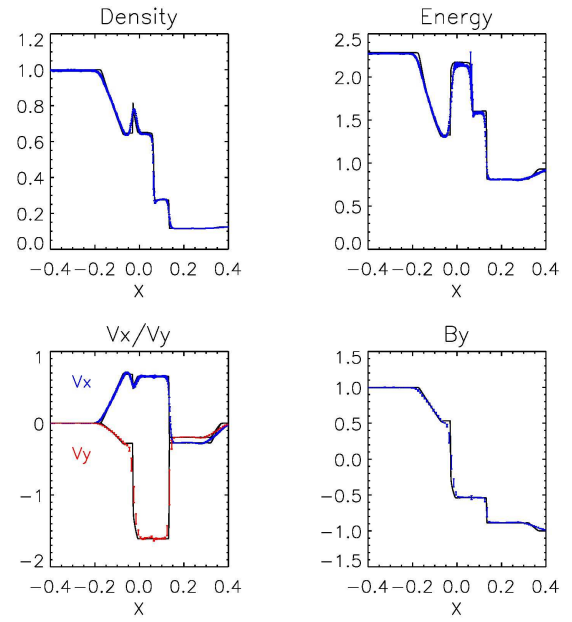

Figure 2: In blue and red the result of different quantities of the Brio and $\mathrm{Wu}[7]$ test compared with the solution from Athena [21] in black line. Compare with Price [17] and Stasyszyn et al. [20], for other solutions.

\subsection{Differentially rotating disk}

A gravitational bound disk with differential rotation is a natural test bed for starting kinematic galactic magnetohydrodynamics and dynamo action. Magnetic field vectors parallel to the velocity vectors are stationary solutions of the induction equation for ideal MHD. Therefore, we setup a rotating disk, following a Brandt profile in the velocity distribution $V_{\phi}(r)=r \Omega_{0} / \sqrt{1+r^{2} / r_{0}^{2}}$, using as parameters for the model $\Omega_{0}=180 \mathrm{Gyr}^{-1}$ and $r_{0}=2.0 \mathrm{kpc}$. We will omit units and interpret length normalized to $1 \mathrm{kpc}$ and time to 1 Gyr. Additionally, we prescribe the static gravitational potential that gives equilibrium between the centrifugal and gravitational forces. Neglecting hydrodynamic forces keeps the rotation constant in time. We use 220949 particles in a disk with radius $R=5$ and height $H=4$. The particles are set up initially equidistant in radius, height and polar angle but with a random phase distribution. Periodic boundary conditions are applied. We start with only a toroidal magnetic field $B_{\phi}$, that is the result of a vector potential $A_{z}=0$ if $r<1, A_{z}=-0.1 \cdot(r-1.0)$ if $1<r<2$ and $A_{z}=-0.1$ if $r>2$. Because magnetic field vectors parallel to the velocity vectors are stationary solutions of the induction equation for ideal MHD, the field should not change during time evolution.

This simple example was numerical unstable for the direct implementation of the magnetic field but stable for the vector potential. The instability is due to the creation of a small radial magnetic field component from the toroidal field by the discretization error. Together with the rotational shear (amplifying again the toroidal field) this leads to an exponential growth of the field with $\nabla \cdot \mathbf{B} \neq 0$. The problematic transfer of errors between components happens because the term $(\mathbf{B} \cdot \nabla) \mathbf{v}$ is used in Eq. (1) instead of the advection term $(\mathbf{v} \cdot \nabla) \mathbf{B}$. The cleaning does not help here, because it does not remove the $B_{r}$ instead it adds a $B_{z}$, which indeed reduces the $\nabla \cdot \mathbf{B}$, but the total field still growth spuriously by the shear and could misleadingly interpreted as dynamo action.

In Fig. 1 we plot the evolution of the mean magnetic field, in dashed black lines the vector potential implementation and in solid red the direct induction formulation. In the lower panel of Fig. 1 we show the evolution of the mean divergence error $\operatorname{Err}(\nabla \cdot \mathbf{B})=h \nabla \cdot \mathbf{B} /|\mathbf{B}|$. In the case of the direct induction we observe an exponential growth, while during the integration with the vector potential the small error related 


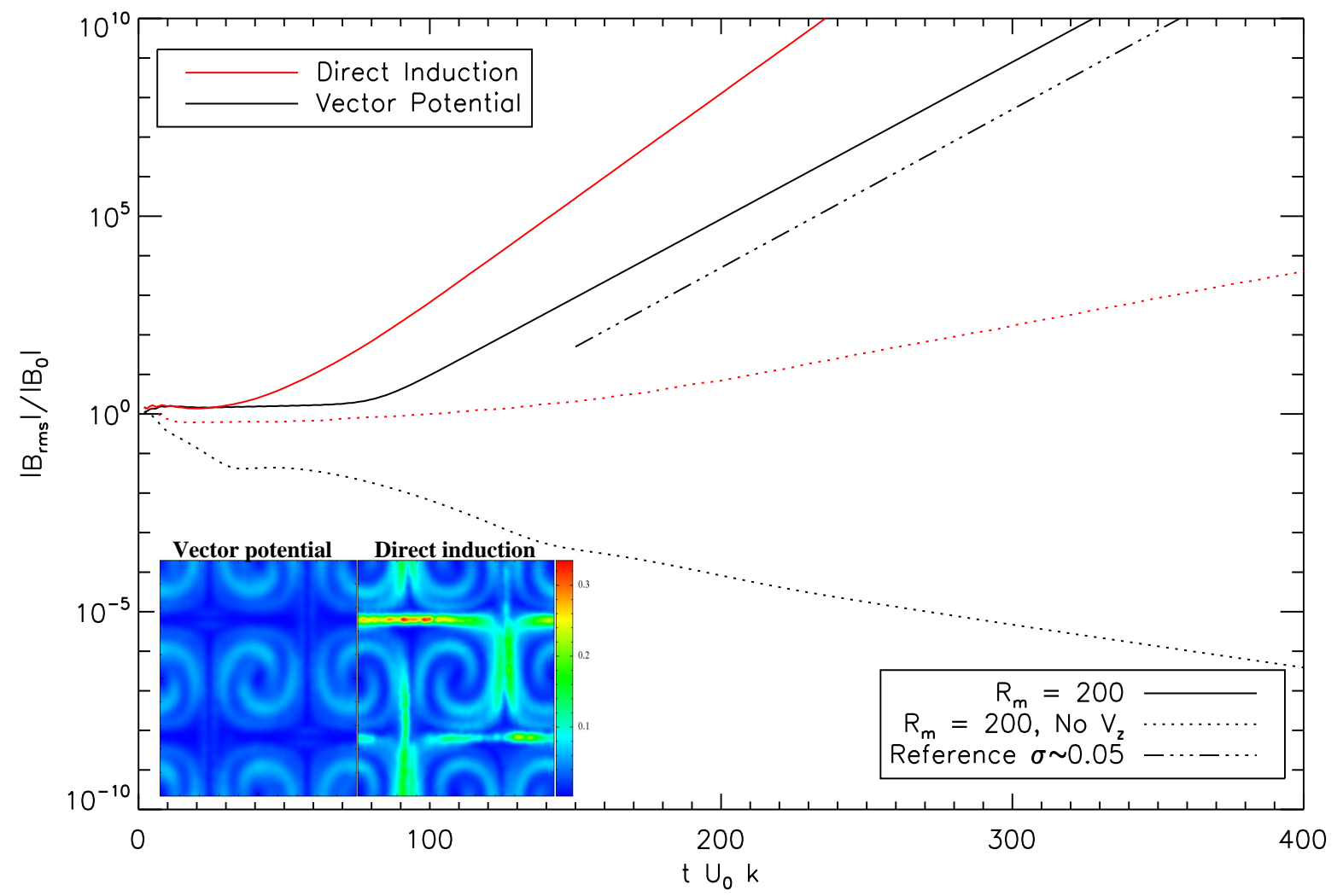

Figure 3: Evolution of the mean magnetic energy density for a Roberts flow $R_{m}=200$ in different cases. We recover the expected growth rate $\sigma \sim 0.05$ for the vector potential, as well the decay for the planar flow $\left(V_{z}=0\right)$. The growth rate is larger in the direct induction implementation and a wrong growth of the magnetic field appears in the planar case. Additionally, we show 2 cuts of the magnetic field strength for the $V_{z}=0$ at early times, where we already can appreciate differences in the evolution. In the dynamo case we do not see major morphological differences.

to the accuracy of the curl and div operator remains constant.

\subsection{Roberts Flow}

We use the Roberts flow to demonstrate the viability of the code to handle basic dynamo case. The periodic flow is set-up with a flow in the form

$$
\mathbf{V}=U_{0}\left[-\sin (k y) \cos (k x), \sin (k x) \cos (k y),(\sqrt{2})^{-1} \cos (k y) \cos (k x)\right]
$$

being $U_{0}$ the characteristic velocity of the flow, $k=2 \pi / L$ and $L$ is the size of the periodic box. Following Brandenburg [5] we set up the problem with magnetic Reynolds number $R_{m}=v k^{-1} \eta^{-1}=200$, which gives a growth rate $\sigma \sim 0.05$. In particular, we use a $U_{0}=16 \pi$ and $\eta=0.04$, in code units. To be able to do this test in SPH we set up a cubic lattice with $50^{3}$ isothermal gas particles of sufficient temperature by prescribing the sound speed $c_{s}=55$, which gives a maximum mach number $M=0.9$. We let the system evolve with the velocity prescribed by the definition Eq. (13) and seed some magnetic field (defined from the vector potentials ${ }^{2}$ in both cases) after a small relaxation time, when the particle distribution stabilises. As

\footnotetext{
${ }^{2}$ In particular we use a vector potential defined as $A_{x}=0, A_{y}=\left(\begin{array}{lllllll}2 & k\end{array}\right)^{-1} \sin (2 k \quad y) \cos (2 k \quad z)$ and $A_{z}=$ $(2 k)^{-1} \sin (2 k z) \cos (2 k y)$
} 
a complement, we run tests setting the vertical velocity $V_{z}=0$. In Fig. (3) we show the resulting evolution of the mean magnetic energy density in the box for the different cases, inlaid two cuts of the magnetic field at early stages for the $V_{z}=0$ case. The ones with a three dimensional velocity field develops a dynamo independent of the implementation and with similar morphologies (therefore not shown). However, the growth rate in the direct induction case is larger than expected. In the planar case, we only have decaying solutions for the magnetic field. The direct induction case shows a wrong growth of the magnetic field. The reason seems to be the wrong advection of the field in curved flows similar to the example of section 3.1. This shows that only the scheme with the vector potential converges to the correct solution.

\subsection{Brio- Wu shock tube}

Basically, we use the same glass like tube setup as described in Dolag and Stasyszyn [10], but enlarging it 10 times in the z-direction, by replicating the initial conditions in that direction. We set up the initial vector potential as $A_{z}=\left|x-L_{X} / 2.\right|$ and $A_{y}=-0.75 z$, where $L_{x}$ is the longitude of the tube domain in the $x$ dimension and we use a total of 350000 particles. The particular definition of $A_{y}$ is the reason why we extend the shock dimension in $z$ to have good spatial derivatives. In the code we take care that the periodic boundary conditions are well fulfilled (particularly in $z$ ). Note that we do not use an external field as Price [17] for the $B_{x}$ component. In Fig. 2 we show in blue the result of the test for the different quantities compared with the solution from Athena [21] in black line. Overall, we found a good agreement between the solutions and previous SPMHD implementations. However there is a noticeable smoothing of the magnetic field, which is expected from the scheme itself.

\subsection{Orzang-Tang Vortex}

This test was introduced by Orszag and Tang [14] and has the complexity of many kinds of interacting waves. We use the same initial conditions as described in Stasyszyn et al. [20]. We configure the vector potential as $A_{z}=(\pi \sqrt{4 \pi})^{-1}[0.5 \cos (2 \pi y)+0.25 \cos (4 \pi x)]$. Price [17] uses a similar procedure and reports tensile instabilities when running in 3 dimensions. We also observe instabilities at $t \approx 0.4$ even with smoothing of the magnetic field, but we are able to avoid them by keeping track of the gauge. At later times $(t>0.6)$ instabilities rise again which is consistent with Candelaresi et al. [8], and on this time we are able to handle them with a little bit of diffusion (see section 2.2 and using a value in code units of $\eta=0.0001$ 3 ). Therefore, we are able to run the test until late times (i.e. $t>6.0$ ) without any problems. Note that this test is usually presented only up to $t \approx 0.5$. The effect of the small dissipation does not trigger any instabilities (see also the discussion in Stasyszyn et al. [20]).

In Fig. 4, we show the density distribution for two different times, $t=0.5$ (which is the one usually used to compare between codes) and $t=1.0$. The solutions found are completely comparable between them and with other methods. In Fig. 5, we show the evolution of the magnetic energy density for these different implementations. They match quite well and the symmetry of the problem is overall maintained.

\section{Implications}

The sole fact that the usual implementation fail in the simple test of section 3.1 is worrisome. The code integrates all the variables in cartesian coordinates, which is not optimal for our cylinder symmetric test with a dominant rotational velocity. But Eulerian grid methods can handle this problem easily in cartesian coordinates. Rotational objects are a common feature of astrophysical simulations and should be traced in a stable and consistent scheme. We cannot rely on the fact that the stochastic approach of the method can cancel out all the errors, but this should be demonstrated. In particular, this test points to the danger to confuse a numerical instability with a physical dynamo instability. Therefore it should be taken into account in the interpretation of the results of the simulations. We exemplify the problem with an astrophysical model of a galaxy.

\footnotetext{
${ }^{3}$ The dissipation value, is chosen to have diffusive times larger enough, compared with other timescales in the problem.
} 


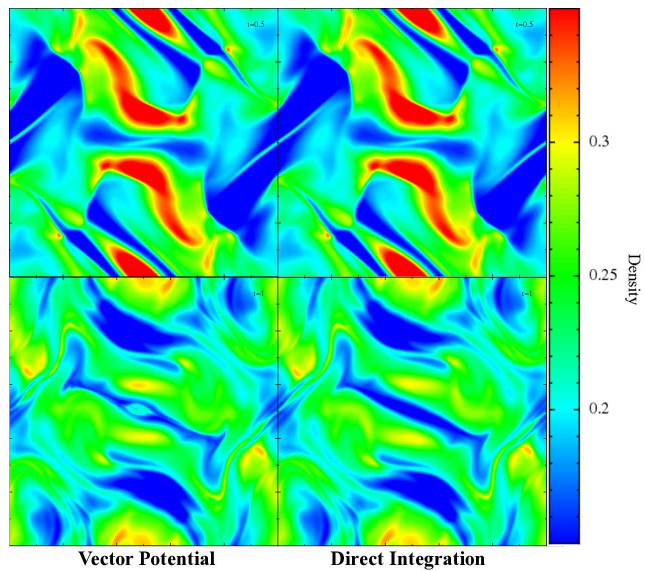

Figure 4: Shown are the density for the Orzang-Tang vortex at time $t=0.5$ (upper panels) and $t=1.0$ (lower panels). The solutions are completely comparable with previous methods.

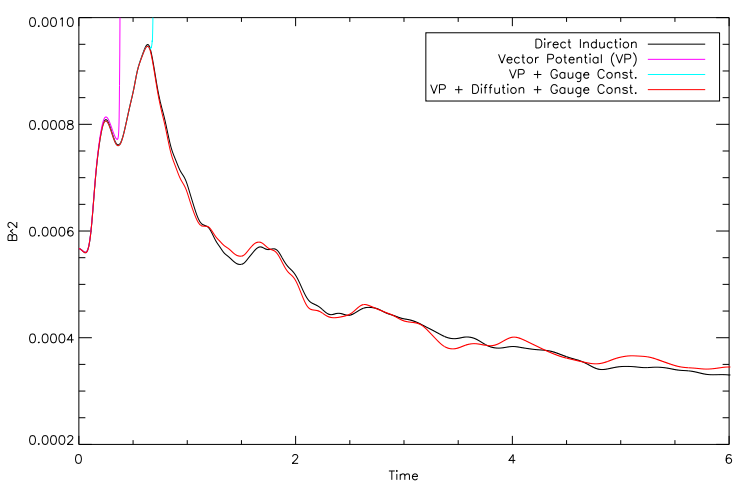

Figure 5: Evolution of the mean magnetic energy density for the Orzang-Tang vortex for the SPH induction implementation and the vector potential. The introduction of the numerical corrections avoid instabilities, not showing noticeable differences affecting the evolution.

\subsection{Example: A Galaxy}

To corroborate possible implications of the methods, we compare both methods in an astrophysical scenario with a fully 3 dimensional flow. We set up a small galaxy, with $3.9 \times 10^{4} \mathrm{SPH}$ particles in disk and IGM, and $3.4 \times 10^{5} \mathrm{DM}$ particles.

The SPH particles are distributed in a disk and inter-stellar medium to avoid spurious effects from the boundary [11]. The total mass of the galaxy is $2.4 \times 10^{11} \mathrm{M} \odot$ and the initial radius of the disk is $r_{0}=20 \mathrm{kpc}$. We let the galaxy evolve during $0.2 \mathrm{Gyr}$, to allow the particles to relax and stabilize, after this time we seed a magnetic field in the disk by a ring of $B_{\phi}=10^{-12} \mathrm{G}$ calculated using only a vector potential in the $A_{z}$ component in the same way as described in section 3.1. We also try a constant $B_{x}$ as initial magnetic configuration with similar results. We evolve the galaxy with both schemes using a small constant dissipation $\eta=6 \times 10^{24} \mathrm{~cm}^{2} / \mathrm{sec}$. Again the direct integration of the magnetic field shows a strong growth of the magnetic energy in contrast to the potential method, where the field slowly decays (cf. Fig. 6). We consider also the dynamical cases, taking into account the Lorentz force.

In the upper panel of Fig. 6, we show the evolution of the mean magnetic energy for the different methods. In red we plot the direct induction and the vector potential in black. Also, solid lines are the result without taking into account the Lorentz force and in dot dashed the full MHD implementation. In the beginning of the simulation both methods seem to agree, afterwards there is a growth to $\mu \mathrm{G}$ levels in the induction case. In the lower panel we show the log absolute value of the average for the different components in cylindrical coordinates normalized to the initial $B_{\phi}$ for an initial subset of the time evolution, until 0.1 Gyr. This is shown only for the kinematic case, therefore the gas dynamics is the same, but the magnetic fields evolution differ. The direct integration leads to a strong numerical growth of the poloidal magnetic field components $B_{r}$ and $B_{z}$ in a similar way as found in the test problem of section 3.1.

For the dynamical simulations, we show density cuts in Fig. 7 with their respective magnetic field vectors, which illustrate the difference caused probably by the unphysical growth of the magnetic field for the direct induction algorithm. The magnetic field remains weak with no dynamical influence on the gas for the solution with the vector potential in contrast to the direct integration of the induction equation with locally 6 orders of magnitude larger field strength. The density is in the same log scale, showing that in the induction case the magnetic pressure prevents the accretion of gas, meanwhile in the vector potential case, we reach higher densities and stronger spiral arms, and there is almost no difference in the evolution with the kinematic case.

Kotarba et al. [13] found a similar behavior for a comparison between Euler potentials and direct induc- 

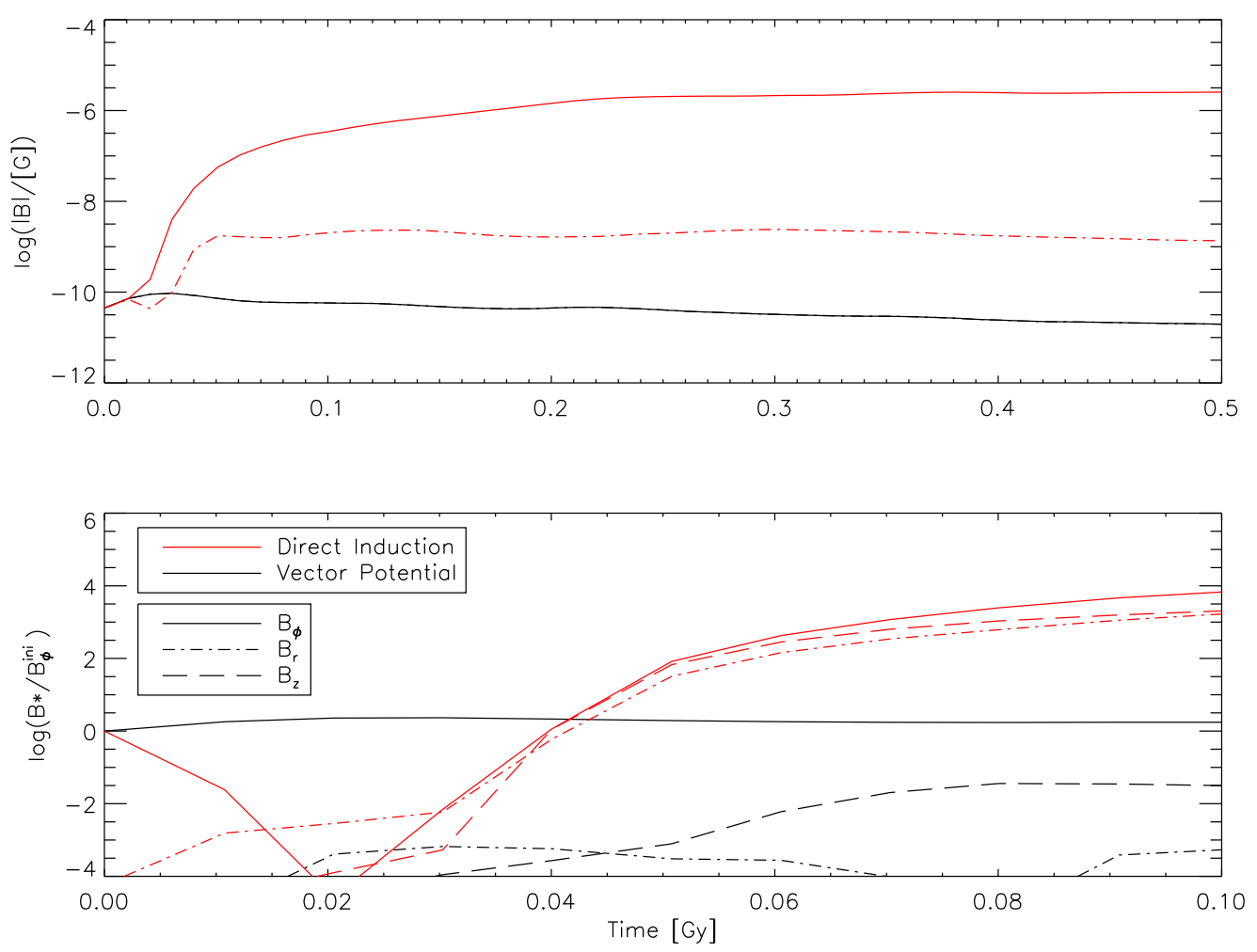

Figure 6: In the upper panel we show the evolution of the mean magnetic field strength for the different methods (red - direct integration, black - vector potential). Solid lines represent the result of the kinematic case (without Lorentz force) and dot dashed lines of the full MHD implementation. In the lower panel we show the evolution of the absolute value of the different components averaged in cylindrical coordinates and normalized to the initial $B_{\phi}$ (solid line is $B_{\phi}$, long dashed is $B_{z}$ and dot dashed is $B_{r}$ ) for the kinematic case.

tion implementations applied to an isolated galaxy. In that case, a stronger growth of the magnetic field in the center of the galaxy was found for the direct induction case when compared to the Euler potential, which is consistent with our results. Kotarba et al. [13] justify the difference in terms of $\nabla \cdot \mathbf{B}$ errors, and discard the full reliability of the Euler implementation because of the fact that the winding up of the field is not correctly traced by the Euler potentials while they are just advected with the velocity field. This is also demonstrated by Stasyszyn et al. [20], in which the Orszag and Tang [14] vortex is evolved several winding up times, and the system evolved through Euler potentials turn out to be unstable at late times. In our case, the vector potentials are fully evolved, which is found to be reliable even in a galaxy scenario.

\section{Conclusions}

This work was triggered by the failing tests of the induction equation, which are naturally solved with the vector potential formalism. This example demonstrates that a divergence error cleaning method does not guarantee to recover the consistent soleonidal solution. We were able to build a vector potential implementation of the SPMHD equations, that successfully passes test cases and an astrophysical scenario. However, more testing and studies are needed, but escapes the scope of the current exploratory work.

In summary, we evolve the vector potential, from which we calculate the magnetic field. We smooth the resulting magnetic field to regularize it and avoid tensile instabilities in the force calculation. We found that 


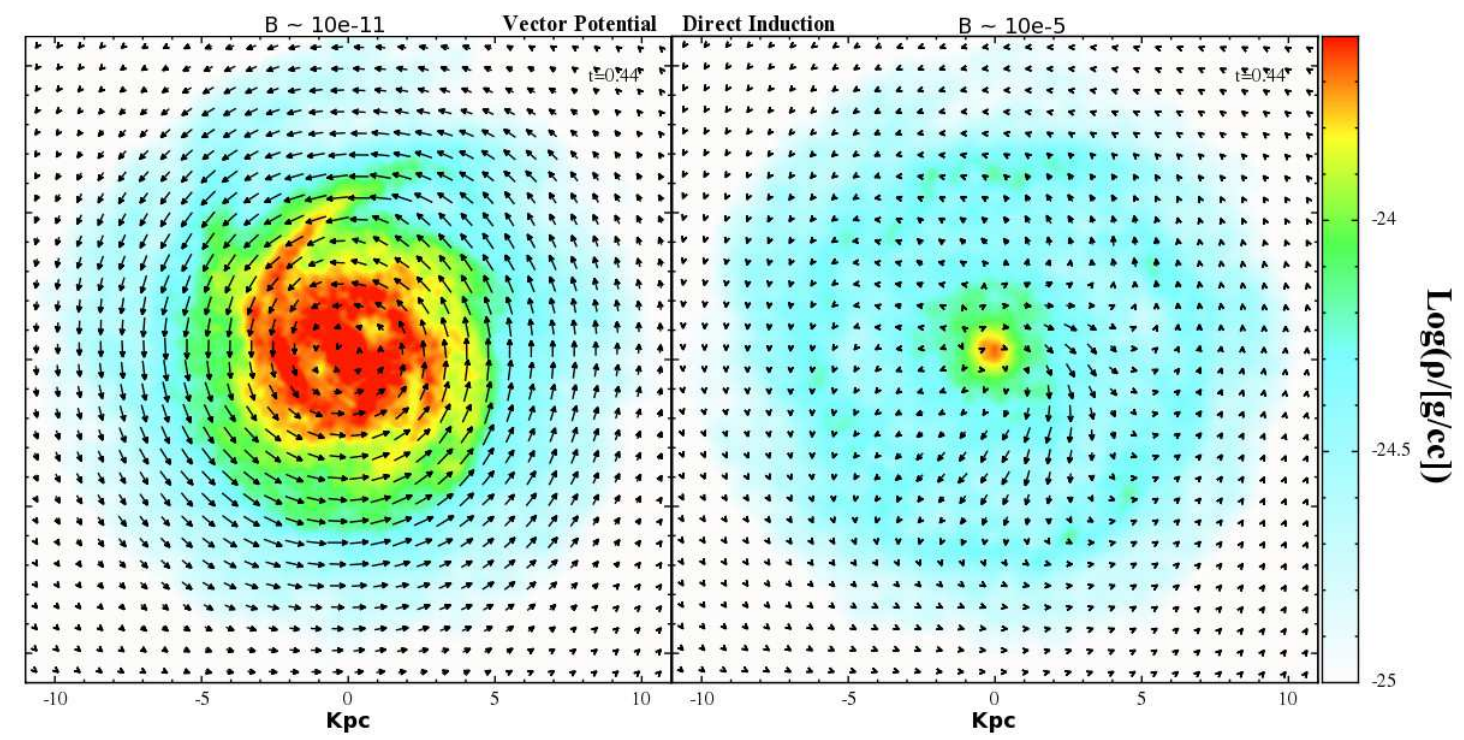

Figure 7: Density cuts through the disk of the galaxy in the dynamic simulations with vector potential (left) and direct integration of the magnetic field (right). Superimposed are the magnetic field vectors normalized to $10^{-11} G$ and $10^{-5} G$, respectively.

the constraint of a correct evolution of the gauge is also important, and we implement a solution similar to the divergence cleaning from Stasyszyn et al. [20] but applied to the vector potential field to be consistent with a pseudo-Coulomb gauge. There are still several possibilities for the gauges, that could be studied in the future. The smoothing of the field and the special care of the gauge in the potential evolution seem to be the key points for which previous implementations failed. Additionally, we implement an explicit diffusion to the equation of the vector potential. This helps to stabilize tests, allowing for example to run the Orszag and Tang [14] vortex up to at least 6 winding times. The kinematic dynamo of the Roberts flow gave the expected growth rate for a $3 \mathrm{D}$ velocity field and a finite magnetic diffusivity, as well in the planar case. The same test fail in both cases for the direct induction with growing solutions.

The comparison of both methods for the astrophysical application of the galaxy evolution shows a similar behavior as the simple rotating disk example. A probably unphysical growth of the initial toroidal magnetic field appears only for the direct integration, while the vector potential method leads only to a radial redistribution of the toroidal field.

Solving directly the induction equation in SPH has been applied in the past. We understand that the standard implementation of the induction does not advect correctly the field in some cases. Such effect has an unclear net effect in the stochastic motions of astrophysical simulations, being possible to be washed out, or not.

The use of the hybrid approach in order to couple the dynamics and evolution of the magnetic field from the vector potential opens space to improve the numerical implementation. The same has to be said for the additional dissipative switches, energy conservation and a deeper study of the method in several environments, which already have been performed in the case of the direct induction evolution in SPMHD. However, the implementation presented here is already robust enough for further applications investigating dynamo processes in astrophysics.

\section{Acknowledgements}

We thank Klaus Dolag for the useful discussions and the referees for their comments that helped to improve this work. For the revision of the text we are grateful to Creasey P. and Lagos C. and Paz D, and comments of the Roberts flow form Candelaresi S. and Del Sordo F. Part of this work was supported by the 
Deutsche Forschungsgemeinschaft (DFG), project number FOR1254. Figure 3 and 6 have been done using SPLASH [16].

\section{References}

[1] Beck, R., Oct. 2009. Galactic and extragalactic magnetic fields - a concise review. Astrophysics and Space Sciences Transactions 5, 43-47.

[2] Beck, R., Brandenburg, A., Moss, D., Shukurov, A., Sokoloff, D., 1996. Galactic Magnetism: Recent Developments and Perspectives. ARA\&A34, 155-206.

[3] Bonafede, A., Dolag, K., Stasyszyn, F., Murante, G., Borgani, S., Dec. 2011. A non-ideal magnetohydrodynamic GADGET: simulating massive galaxy clusters. MNRAS418, 2234-2250.

[4] Børve, S., Omang, M., Trulsen, J., Nov. 2001. Regularized Smoothed Particle Hydrodynamics: A New Approach to Simulating Magnetohydrodynamic Shocks. ApJ561, 82-93.

[5] Brandenburg, A., Jan. 2010. Magnetic field evolution in simulations with Euler potentials. MNRAS401, 347-354.

[6] Brandenburg, A., Käpylä, P. J., Aug. 2007. Magnetic helicity effects in astrophysical and laboratory dynamos. New Journal of Physics 9, 305.

[7] Brio, M., Wu, C. C., Apr. 1988. An upwind differencing scheme for the equations of ideal magnetohydrodynamics. Journal of Computational Physics 75, 400-422

[8] Candelaresi, S., Hubbard, A., Brandenburg, A., Mitra, D., Jan. 2011. Magnetic helicity transport in the advective gauge family. Physics of Plasmas 18 (1), 012903.

[9] Dedner, A., Kemm, F., Kröner, D., Munz, C.-D., Schnitzer, T., Wesenberg, M., Jan. 2002. Hyperbolic Divergence Cleaning for the MHD Equations. Journal of Computational Physics 175, 645-673.

[10] Dolag, K., Stasyszyn, F., Oct. 2009. An MHD GADGET for cosmological simulations. MNRAS398, 1678-1697.

[11] Geng, A., Kotarba, H., Bürzle, F., Dolag, K., Stasyszyn, F., Beck, A., Nielaba, P., Feb. 2012. Magnetic field amplification and X-ray emission in galaxy minor mergers. MNRAS419, 3571-3589.

[12] Jackson, J. D., Sep. 2002. From Lorenz to Coulomb and other explicit gauge transformations. American Journal of Physics 70, 917-928.

[13] Kotarba, H., Lesch, H., Dolag, K., Naab, T., Johansson, P. H., Stasyszyn, F. A., Aug. 2009. Magnetic field structure due to the global velocity field in spiral galaxies. MNRAS397, 733-747.

[14] Orszag, S. A., Tang, C., Jan. 1979. Small-scale structure of two-dimensional magnetohydrodynamic turbulence. Journal of Fluid Mechanics 90, 129-143.

[15] Phillips, G. J., Monaghan, J. J., Oct. 1985. A numerical method for three-dimensional simulations of collapsing, isothermal, magnetic gas clouds. MNRAS216, 883-895.

[16] Price, D. J., Oct. 2007. splash: An Interactive Visualisation Tool for Smoothed Particle Hydrodynamics Simulations. Publications of the Astronomical Society of Australia 24, 159-173.

[17] Price, D. J., Jan. 2010. Smoothed Particle Magnetohydrodynamics - IV. Using the vector potential. MNRAS401, 14751499.

[18] Price, D. J., Feb. 2012. Smoothed particle hydrodynamics and magnetohydrodynamics. Journal of Computational Physics 231, 759-794.

[19] Ruzmaikin, A. A. and Sokolov, D. D. and Shukurov A.M. (Ed.), 1988. Magnetic fields of galaxies. Vol. 133 of Astrophysics and Space Science Library.

[20] Stasyszyn, F. A., Dolag, K., Beck, A. M., Jan. 2013. A divergence-cleaning scheme for cosmological SPMHD simulations. MNRAS428, 13-27.

[21] Stone, J. M., Gardiner, T. A., Teuben, P., Hawley, J. F., Simon, J. B., Sep. 2008. Athena: A New Code for Astrophysical MHD. ApJS178, 137-177.

[22] Tricco, T. S., Price, D. J., Aug. 2012. Constrained hyperbolic divergence cleaning for smoothed particle magnetohydrodynamics. Journal of Computational Physics 231, 7214-7236.

[23] Widrow, L. M., 2002. Origin of galactic and extragalactic magnetic fields. Reviews of Modern Physics 74, 775-823. 